\title{
Cytoskeletal responsiveness to progestins is dependent on progesterone receptor A levels
}

\author{
E M McGowan, R P Weinberger'1, J D Graham, H D Hill, J A I Hughes', \\ G M O'Neill' ${ }^{1}$ and C L Clarke
}

Westmead Institute for Cancer Research, University of Sydney at the Westmead Millennium Institute, Westmead Hospital, Westmead, New South Wales 2145, Australia

${ }^{1}$ Oncology Research Unit, The Children's Hospital at Westmead, New South Wales 2145 and the Department of Pediatrics and Child Health, University of Sydney, New South Wales 2006, Australia

(Requests for offprints should be addressed to C L Clarke; Email: christine_clarke@wmi.usyd.edu.au)

(E M McGowan and R Weinberger contributed equally to this work)

\begin{abstract}
Changes in the cell cytoskeleton occur in cell transformation and recent data suggest the involvement of ovarian hormones, which are implicated in cancer development and progression. In human breast and endometrial tumors, there is disrupted expression of progesterone receptor (PR) isoforms and predominance of one isoform, usually PRA. PRA predominance is an early event in carcinogenesis, and in cancers is associated with poor clinical features. Overexpression of PRA in vitro causes altered progestin regulation of cell morphology, suggesting that PRA overexpression may provoke deleterious changes in cell functioning. This study aimed to identify pathways of cytoskeleton regulation responsive to progestins and to determine whether these are perturbed when PRA is overexpressed to the levels seen in cancers. Progestin treatment of PR-positive breast cancer cells caused increased cell surface area whereas after induction of a stably integrated PRA construct, cells became rounded and the cell surface was decreased. The effect of PRA induction on cell rounding was reversed by the anti-progestin RU38486. Altered tropomyosin $(\mathrm{Tm})$ isoforms were implicated in these morphological differences, as there was a PRA-mediated alteration in Tm5 isoform levels, and transfection of Tm5a mimicked progestin-mediated cell rounding in PRA-overexpressing cells. Ezrin was redistributed from the membrane to cytoplasmic locations in the presence of progestin, and discrete focal localization was evident in cells with PRA predominance. Progestin effects on the cytoskeleton in PRA-overexpressing cells provide evidence for novel endocrine regulation of aspects of actin microfilament composition, suggesting that changes in the cytoskeleton known to be associated with cancer development and progression may be regulated in part by altered PRA expression which develops early in carcinogenesis.
\end{abstract}

Journal of Molecular Endocrinology (2003) 31, 241-253

\section{Introduction}

There is well-described epidemiological, experimental and clinical evidence that ovarian hormones play a major role in the development and progression of female hormone-dependent cancers. Women without ovaries have a very low risk of breast and endometrial cancer, whereas cumulative exposure to ovarian hormones, particularly estrogen, is associated with increased risk (MacMahon \& Feinlieb 1960). There is also evidence that women exposed to exogenous progesterone have increased breast cancer risk (Ross et al. 2000) and a recent randomized trial showed increased breast cancer risk in healthy postmenopausal women on combined estrogen-progestin hormone replacement therapy (Writing Group for the Women's Health Initiative Investigators 2002). This is supported by evidence that ablation of nuclear progesterone receptor $(\mathrm{PR})$ decreases mammary carcinogenesis in mice (Lydon et al. 1999), and suggests that progesterone may play a role in breast cancer development.

The mechanisms of progesterone involvement in breast cancer development are unknown, but there are recent data which have provided evidence for 
disrupted progesterone signaling in human breast and endometrial tumors. The nuclear PR is expressed as two proteins: PRB and PRA (Kastner et al. 1990), which have different transcriptional activities (Gronemeyer 1991, Tung et al. 1993, Vegeto et al. 1993, McDonnell et al. 1994, Hovland et al. 1998, McGowan \& Clarke 1999). PRA and PRB are co-expressed, at similar levels, in normal breast (Mote et al. 2002) and endometrial epithelial cells (Mote et al. 1999), implicating both isoforms in the known effects of progesterone in these tissues. Progression to malignancy in breast and endometrium is accompanied by progressively unequal expression of PRA and $\mathrm{PRB}$, and a significant proportion of carcinomas express a predominance of one isoform, usually PRA (Arnett-Mansfield et al. 2001, Mote et al. 2002). This is associated with poor clinical outcomes: in endometrial cancer, PR isoform predominance, usually of PRA, is significantly more common in tumors of higher grade, indicating an association between PR isoform predominance and poor prognosis (ArnettMansfield et al. 2001). The loss of co-ordinated PRA and PRB expression is an early event in carcinogenesis and is evident in early pre-malignant lesions (Mote et al. 2002). The available evidence supports the view that progesterone signaling in normal target epithelial cells requires the concerted activity of co-expressed PRA and PRB, and that disruption of PRA and PRB expression results in altered biological features which may play a role in hormone-dependent cancers.

In vitro studies aimed at asking whether PRA overexpression seen in cancers is a consequence of the carcinogenic process, or plays a role in its development and/or progression have revealed that overexpression of PRA results in altered progestin regulation of cell morphology, suggesting that development of PRA overexpression may itself provoke deleterious changes in cell functioning. In cells overexpressing the PRA isoform but not in control cells, cells acquired rounded morphology and there was decreased adherence of cells to tissue culture flasks (McGowan \& Clarke 1999). This suggests that the cell cytoskeleton is a target of progesterone action, particularly when PRA predominates, and, as cell rounding and loss of adhesion are hallmarks of invasive potential, that the endocrine regulation of the cytoskeleton may be implicated in hormone-dependent cancer.
The cytoskeletal system most critical in transducing information from the cellular environment is the actin-tropomyosin (Tm) microfilament system (Hall 1998), which is closely linked to morphological changes that occur within cells during development (Gunning et al. 1998b) and in the transformed phenotype (Leavitt et al. 1987a,b). Changes in the microfilament system have been linked with cell transformation and tumorigenicity (Leavitt et al. 1987a,b). Microfilament bundles or stress fibers are commonly reduced in size and number and show altered organization after transformation (Pollack et al. 1975). Disruption of stress fibers has been shown to correlate with other features of the transformed phenotype, including loss of contact inhibition, acquisition of anchorageindependent growth, and tumorigenicity (Edelman \& Yahara 1976, David-Pfeuty \& Singer 1980). Tumor promoters alter the cytoskeleton, demonstrating a relationship between the cytoskeleton and the normal mechanisms of cellular proliferation control (Takahashi et al. 1986, Pienta et al. 1989).

The mechanisms underlying the changes in the cytoskeleton that occur in cancer are not fully understood; however, in light of the progestin effects on the cytoskeleton in cells overexpressing PRA, it is likely that cross-talk between the cytoskeleton and endocrine signaling may be involved. The purpose of this study was to determine whether the actin-Tm microfilament system was regulated by progestins and to determine whether overexpression of PRA, which is an early event in carcinogenesis of hormonedependent tissues, alters the progestin effects on these cytoskeletal components. The overall aim of these studies is to identify cellular signaling pathways which may lead to changes in cell morphology in response to hormones and contribute to poorer prognosis phenotypes in hormonedependent cancers.

\section{Materials and Methods}

\section{Plasmids and antibodies}

Rat Tm5a and Tm5b cDNAs were excised from the $\beta$-actin expression vector (Qin \& Gunning 1997) and cloned into the SalI site of the pHßApr-4(sig-) vector (pH $\beta$ Apr-4Tm5a and pHßApr-4Tm5b). Monoclonal antibodies against human PR (hPRa6 and 7) were isolated from 
hybridoma cultures (Clarke et al. 1987). The $\mathrm{ws}_{\mathrm{f}}-9 \mathrm{~d}$ polyclonal antibody, produced in rabbit (Schevzov et al. 1997), detects Tms from exon 9d of the $\alpha-T m_{\text {fast }}$ and $\beta$ - Tm genes, and has been described previously (Percival et al. 2000). Rabbit polyclonal anti-ezrin (Upstate Biotechnology, Waltham, MA, USA), anti-vinculin and anti- $\beta$ actin monoclonal antibodies (Sigma Biosciences, St Louis, MO, USA), and mouse monoclonal $\beta$-catenin antibody (BD Transduction Laboratories, Franklin Lakes, NJ, USA), were used for immunoblots and immunofluorescence.

\section{Cell culture}

The estrogen receptor (ER)-positive, PR-positive T-47D cells (Keydar et al. 1979) (E G Mason Research Institute, Worcester, MA, USA) were cultured in antibiotic-free phenol red-free RPMI 1640 medium and supplemented as described (Graham et al. 1995a). T-47Dp3'SS clones containing the p3'SS vector, encoding the lac repressor, and the T-47DhPRA cell lines containing both the p3'SS and inducible pOP13 hPRA plasmids were maintained in medium supplemented with hygromycin B (200 $\mu \mathrm{g}$ / $\mathrm{ml})$ and G418 (200 $\mu \mathrm{g} / \mathrm{ml})$. T-47Dp3'SS, which are similar to wild-type T-47D cells and were used as controls, and T-47DhPRA cell lines have been described previously (McGowan \& Clarke 1999). Cells were negative for mycoplasma contamination as determined using the Genprobe rapid detection system (Gen-Probe, Inc., San Diego, CA, USA). Cell stocks were passaged regularly to maintain exponential growth. For experiments, cells were seeded at a density of $2 \times 10^{4}$ cells $/ \mathrm{ml}$ unless otherwise indicated. Twenty-four hours later (day -1$)$ the medium was supplemented with vehicle or with $10 \mathrm{mM}$ isopropyl- $\beta$-thiogalactosidase (IPTG) (Gibco/BRL, Melbourne, Australia), to sequester the lac repressor and allow induction of PRA from pOP13 hPRA. On day 0 the medium was supplemented with the synthetic progestin ORG2058 (10 nM) (Organon Chemicals, Amersham Australia, Sydney, NSW, Australia) or vehicle only. Where indicated cells were also treated with the anti-progestin RU38486 $(100 \mathrm{nM})$, or estradiol $(10 \mathrm{nM})$, either alone or in combination with ORG2058 (10 nM).

\section{Transient transfections}

T-47DhPRA cells plated on Lab-Tek II RS glass chamber slides (Nalge Nunc International, IL,
USA) at a density of $2 \times 10^{4}$ cells $/ \mathrm{ml}$ were co-transfected with pH $\beta A p r-4 \operatorname{Tm} 5 \mathrm{a}(1 \mu \mathrm{g})$, or pHßApr-4Tm5b (1 $\mu \mathrm{g})$ and pEGFP (500 ng), using the Fugene transfection protocol (BoehringerMannheim, Indianapolis, IN, USA) (GFP= green fluorescent protein). Cells were treated with IPTG $(10 \mathrm{mM})$ or vehicle for $24 \mathrm{~h}$ before treating with ORG2058 (10 nM) or vehicle for a further $72 \mathrm{~h}$. All experiments were performed in triplicate.

\section{Immunoblot analysis}

T-47D p3'SS and T-47DhPRA cells were harvested and stored as described previously (Alexander et al. 1989). Cell pellets were thawed on ice in PEMTG buffer (Graham et al. 1995a) containing $0.4 \mathrm{M} \mathrm{KCl}$ and protease inhibitors $(0.5 \mathrm{mM}$ phenylmethylsulfonyl fluoride, $1.4 \mu \mathrm{M}$ pepstatin A, bacitracin $(100 \mu \mathrm{g} / \mathrm{ml}), 25 \mathrm{mM}$ benzamidine, $86 \mu \mathrm{M}$ leupeptin and aprotinin $(77 \mu \mathrm{g} / \mathrm{ml}))$. Cell extracts were prepared and $25 \mu \mathrm{g}$ protein transferred to nitrocellulose as described (Graham et al. $1995 a$ and references therein). Protein concentration was determined by the method of Bradford (Bio-Rad, Richmond, CA, USA). Blots were incubated with antibodies at saturating concentrations and goat anti-mouse and anti-rabbit immunoglobulins linked to horseradish peroxidase at 1:5000 (Bio-Rad). Immunoreactivity was visualized using a chemiluminescent method (ECL; Amersham Pharmacia Biotech, Piscataway, NJ, USA). The relative intensity, in the linear range of the film, of the immunoreactive bands was calculated after densitometric scanning of X-ray films and analysis using Imagequant software (Molecular Dynamics Inc., Sunnyvale, CA, USA). Results are expressed as the percentage of immunoreactivity compared with control.

\section{Determination of cellular morphologies}

For determination of cell shape after Tm5a or Tm5b transfection, the proportion of transfected (GFP-positive) cells with round morphology was determined as a proportion of all transfected cells in 20 fields at $\times 400$ magnification. For determination of cell surface area, fluorescence and brightfield images of T-47D cells, stained with $\beta$-catenin antibody to delineate cell boundaries, were captured through FITC filters using a SPOTII cooled CGD digital camera (Diagnostic 
Instruments, Inc., MI, USA) and fluorescence was visualized using an Olympus BX50 microscope. The surface area of the cells was measured using ImagePro plus software (MediaCybernetics, Silversprings, Marland, IL, USA) and analyzed using SPSS statistical software (SPSS Inc., Chicago, IL, USA).

\section{Results}

\section{PRA predominance results in altered cell size}

Progestin treatment of breast cancer cells expressing PRA and PRB resulted in growth inhibition and in adoption of a phase-dark, flattened morphology compared with control (Fig. 1A; a, b). Cell surface was greater in progestin-treated cells compared with vehicle-treated cells (Fig. 1B; $P<0.001$, one-way ANOVA). PRB was predominant over PRA in these cells and the ratio of PRA to $\mathrm{PRB}$ was around $0 \cdot 3$ (not shown). Induction of PRA, such that the PRA/PRB ratio increased to 3 , caused no change either in cell morphology or in cell surface compared with control cells (Fig. 1A, e; Fig. 1B; $P=0 \cdot 26$, one-way ANOVA). However, upon treatment with progestin, PRAoverexpressing cells experienced a marked change in cell morphology. Cells were phase-bright and rounded (Fig. 1A; f) and the cell surface was significantly smaller in progestin-treated PRAoverexpressing cells compared with progestintreated controls (Fig. 1B; $P<0.001$, one-way ANOVA). The cell rounding observed on progestin treatment of cells overexpressing PRA was observed in a range of T-47DhPRA clones with different total PR levels (not shown), demonstrating that the effect seen upon PRA induction was not due to an overall increase in PR levels. In support of this, previous studies have shown that PRA predominance resulted in progestin-mediated effects on gene expression which were related not to total PR levels but to the ratio of PRA to PRB (McGowan \& Clarke 1999).

\section{Progestin specificity of cell morphological changes}

The anti-progestin RU38486 decreased cell numbers similarly to progestin (data not shown), but unlike progestin had no effect on cell surface (Fig. 1A: compare panel b with c; Fig. 1B).
RU38486 treatment abrogated the progestinmediated increase in cell surface (Fig. 1A: compare panel b with d; Fig. 1B). Cells overexpressing PRA and treated with RU38486 had the same appearance as control RU38486-treated cells (Fig. 1A: compare panel $\mathrm{g}$ with $\mathrm{c}$ ). However, combined treatment with progestin and RU38486 reversed the progestin-mediated cell rounding and decreased cell surface in PRA-overexpressing cells (Fig. 1A, compare $\mathrm{f}$ and h; Fig. 1B) supporting the conclusion that these effects were mediated via the PR.

To determine whether the changes in cell morphology were specific to progestins when PRA was overexpressed, or whether activation of a related nuclear receptor could also produce cell rounding, cells were treated with estrogen to activate the ER pathway. Cells numbers were increased after $72 \mathrm{~h}$ of treatment with estradiol, as expected in these cells, but there was no alteration in cell morphology (Fig. 2E and F) as was seen in parallel cultures treated with progestin with or without PRA induction (Fig. 2C and D). ER is expressed in the T-47DhPRA N5 clones and this was confirmed by immunoperoxidase staining of cells grown on coverslips (data not shown).

\section{Progestin regulation of focal adhesion complex proteins and $\beta$-catenin expression in T-47DhPRA cells}

To determine whether the reduced surface area caused by progestin in PRA-overexpressing cells was due to altered levels of cell interaction proteins, levels of candidate proteins were measured by immunoblot in T-47D and T-47DhPRA cells. Progestin treatment resulted in the well-described decrease in levels of PRA and PRB proteins (Fig. 3A) and the increased level of PRA after induction with IPTG is evident (Fig. 3A, compare lane 5 and 7). The levels of $\beta$-catenin, ezrin (Fig. 3B and $\mathrm{C}$ ), $\beta$-actin, $\gamma$-actin, paxillin, talin or focal adhesion kinase (not shown) were unaffected by progestin treatment, either in T-47D cells or in control T-47DhPRA cells (Fig. 3B and C, lanes 1-6) or in cells overexpressing PRA (Fig. 3B and C, lanes 7-8). Vinculin levels were increased by ORG2058 treatment in parental and T-47DhPRA cells (Fig. 3D, compare lanes 1 and 2, 3 and 4, 5 and 6) and this increase was unaffected by induction of PRA (Fig. 3D, compare lanes 7 and 8 with 5 and 6). 

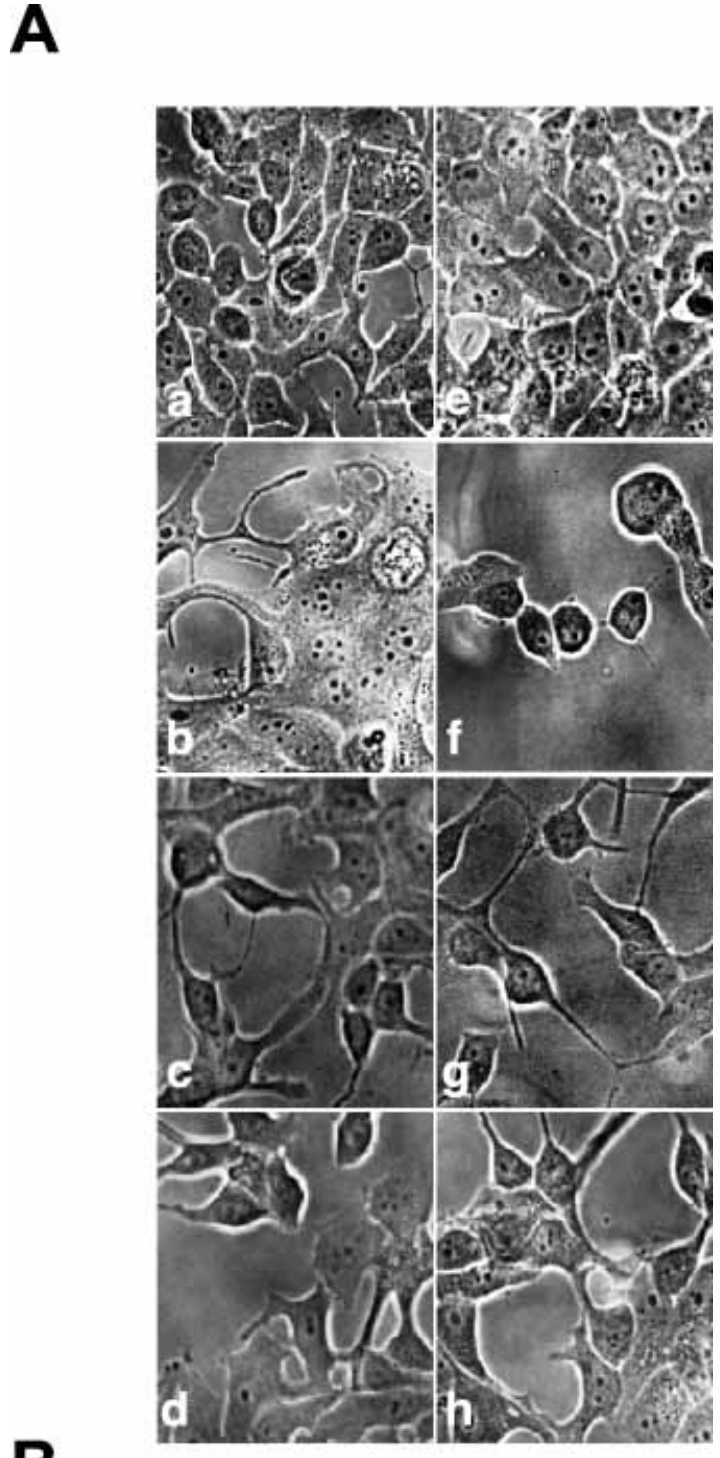

B

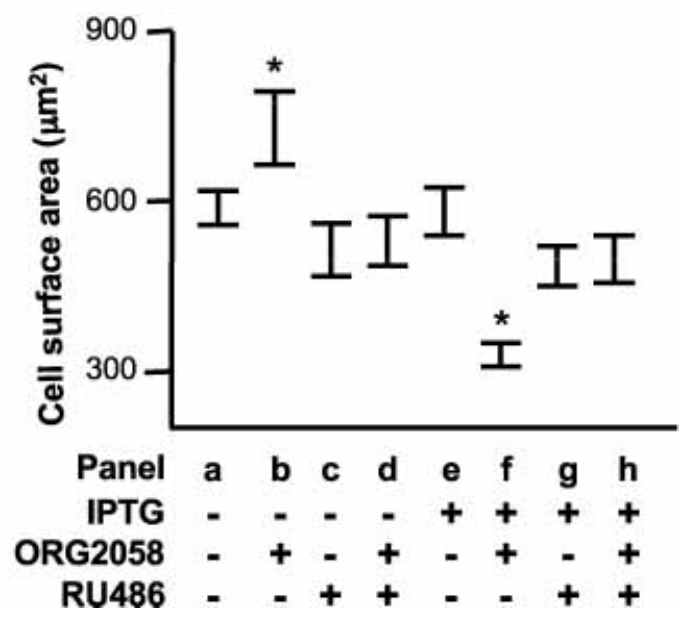

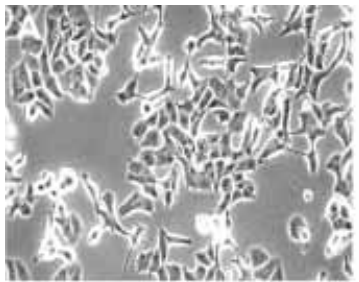
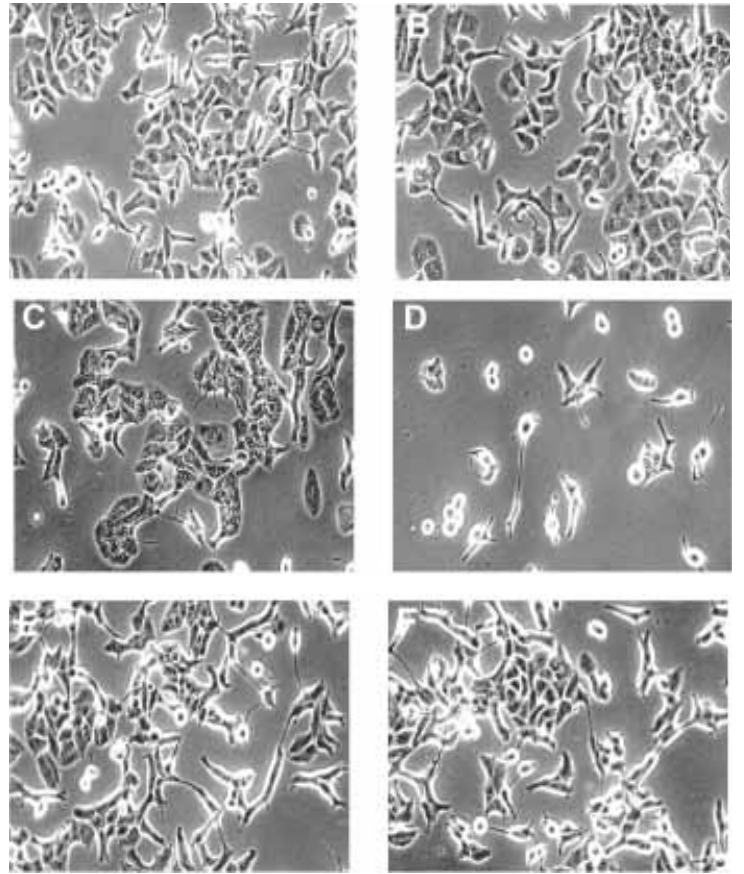

Figure 2 Estrogen effects on cell morphology in T-47DhPRA cells. T-47 DhPRA (clone N5) cells were seeded in $75 \mathrm{~cm}^{2}$ flasks at a density of $5 \times 10^{5}$ cells. Cells were treated with IPTG $(10 \mathrm{mM})$ or vehicle for $24 \mathrm{~h}$ then treated with ORG2058 (10 nM), 17 $(10 \mathrm{nM})$ or their combination. Photographs show the morphology of the cells $72 \mathrm{~h}$ later. (A) vehicle, (B) IPTG (10 mM), (C) ORG2058 (10 nM), (D) IPTG (10 mM) and ORG2058 (10 nM), (E) 17ß-estradiol (10 nM), (F) IPTG $(10 \mathrm{mM})$ and $17 \beta$-estradiol $(10 \mathrm{nM})$. Original magnification $\times 400$.

The progestin induction of vinculin expression was slow and necessitated treatment times of several days to be maximally detectable by immunoblot

Figure 1 Progestin and anti-progestin effects on cell morphology in T-47DhPRA cells. T-47DhPRA cells were seeded in $25 \mathrm{~cm}^{2}$ flasks at a density of $2 \times 10^{4}$ cells $/ \mathrm{ml}$. Cells were treated with IPTG $(10 \mathrm{mM})$ or vehicle for $24 \mathrm{~h}$ then treated with ORG2058 (10 nM), RU38486 (100 nM) or their combination. (A) Photographs a-h show the morphology of the cells $72 \mathrm{~h}$ later. (a) Vehicle, (b) ORG2058 (10 nM), (c) RU38486 (100 nM), (d) RU38486 $(100 \mathrm{nM})$ and ORG2058 (10 nM), (e) IPTG (10 mM), (f) IPTG (10 mM) and ORG2058 (10 nM), (g) IPTG (10 mM) and RU38486 (100 nM), (h) IPTG (10 mM) and RU38486 $(100 \mathrm{nM})$ and ORG2058 (10 nM). Original magnification $\times 400$. (B) The surface areas of cells were measured using ImagePro software. All experiments were performed in triplicate and the data expressed as means \pm S.E.M. *significantly different from control $(P<0.001$, ANOVA). 

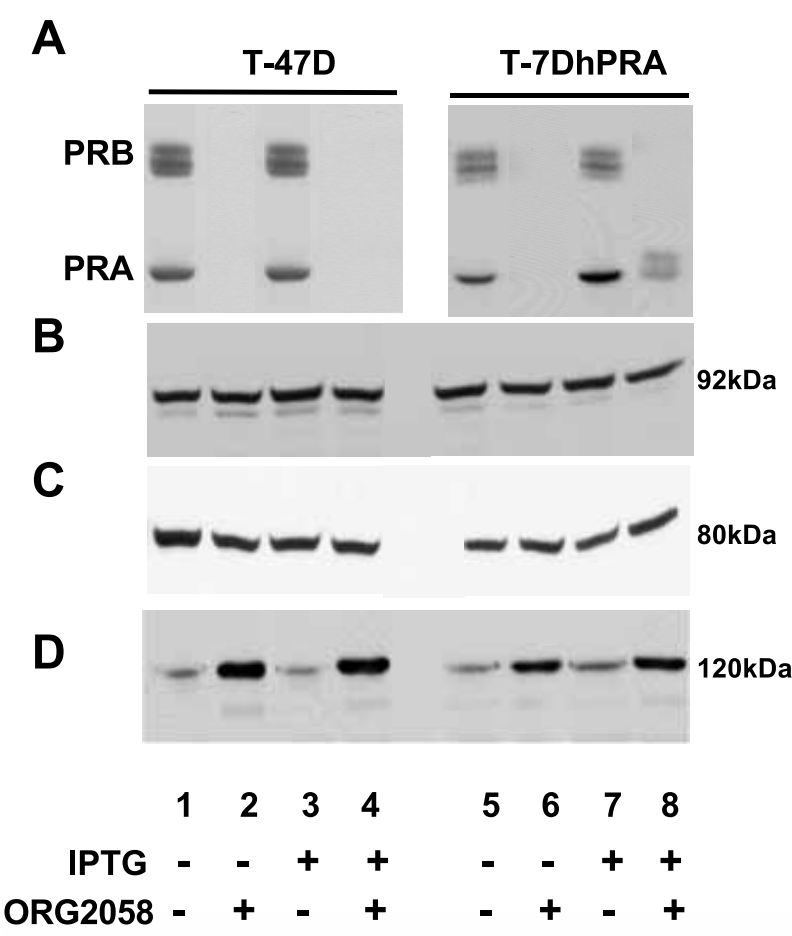

E

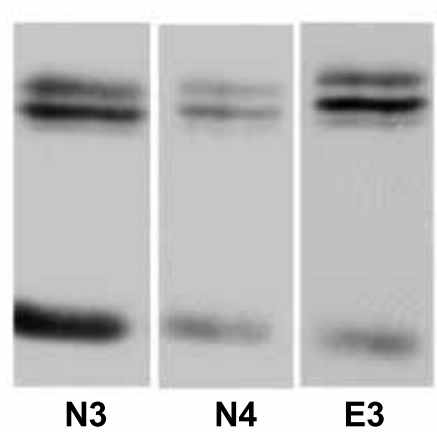

PRB

PRA

Figure 3 Progestin effect on cytoskeletal protein expression in T-47DhPRA cells. T-47DhPRA cells were treated with IPTG $(10 \mathrm{mM})$ or vehicle for $24 \mathrm{~h}$ before treatment with ORG2058 $(10 \mathrm{nM})$ or vehicle. Cells were harvested $72 \mathrm{~h}$ later and total cell protein $(25 \mu \mathrm{g}$ protein/lane) was analyzed by immunoblotting. (A) PRA (94 kDa) and PRB (116 kDa); (B) $\beta$-catenin;

(C) ezrin; (D) vinculin; (E) immunoblot of T-47DhPRA clones N3, N4 and E3. Equal protein levels were loaded onto the immunoblot.

analysis (not shown). Taken together, these data show that although there is progestin regulation of some adhesion proteins, there is no differential effect in control and PRA-overexpressing cells, at least at the level of steady-state protein expression.
This suggests that the differences in progestin effects on cell morphology in cells overexpressing PRA did not result primarily from altered protein levels of membrane components of cell interaction complexes.

The progestin effects on cell surface were observed in clonal T-47DhPRA cell lines with varying PR levels and PRA to PRB ratios. The N3 and E3 clones have abundant PR expression but very different PRA to PRB ratios, which were respectively 1 and $0 \cdot 3$ (Fig. 3E). N4 cells have lower total PR levels with a PRA to PRB ratio close to 1 (Fig. 3E). Induction of PRA with IPTG and progestin treatment in each of these clonal cell lines resulted in the decreased cell surface shown in Fig. 1. The data in Fig. 3E also illustrate the finding that PRB predominance does not result in the cell surface changes seen upon progestin treatment of PRA-overexpressing cells. A number of T-47DhPRA cell lines, illustrated by the E3 clone in Fig. 3E, have a significant predominance of PRB, yet progestin treatment of cell lines with PRB predominance does not result in cell rounding (not shown), whereas progestin treatment after induction of PRA in the same cells causes a clear reduction in cell surface. This is true also for cells with total PRB levels within the range of total PRA levels seen in the cells with induced PRA.

$\beta$-actin and $\gamma$-actin were abundantly expressed in T-47D and T-47DhPRA cells, and no change in the expression levels of either cytoskeletal protein was detectable by immunoblot on progestin treatment of control cells or in cells overexpressing PRA (data not shown).

\section{Regulation by progestin of the actin-Tm family of proteins}

The effects of progestin on the expression of the integral proteins of the microfilament system were determined by immunoblot analysis. Tm isoforms are thought to differentially regulate the biochemical and physical properties of microfilaments: specific Tm isoforms associate in particular microfilaments, which may have distinct cytoskeletal localization and function, and thus control specific features of cell shape (Gunning et al. 1998a). The high molecular weight Tml encoded by the $\beta$-gene was not present in T-47D cells, nor were the Tm2 and Tm6 isoforms, encoded by the 
$\alpha$-gene, detected. Tm3 and the alternatively spliced isoforms, Tm5a and Tm5b (Percival et al. 2000) were detected in T-47D cells. Tm3 was abundant in parental T-47D and in T-47DhPRA cells and its levels were unchanged by progestin treatment or by overexpression of PRA (Fig. 4A). In parental T-47DN and in T-47DhPRAN3 cells, ORG2058 treatment altered the relative expression of Tm5a and Tm5b, preferentially increasing the Tm5b isoform at the expense of the Tm5a isoform (Fig. 4A; compare lanes 1 and 2, and 3 and 4, and 5 and 6). Overexpression of PRA in T-47DhPRA cells diminished the progestininduced increase in the level of Tm5b and this resulted in altered relative levels of Tm5b/Tm5a (Fig. 4A, compare lanes 7 and 8). These changes in Tm5 levels required prolonged exposure to progestins, as they were not evident at $24 \mathrm{~h}$ after treatment (not shown) whereas they were detectable $72 \mathrm{~h}$ after treatment. The progestin effects on relative Tm5 isoform levels was seen in all T-47DhPRA clones tested (hPRA inducible clones with different total PR levels: N3, B8 and E3 and their parental N, B and E cells lacking PRA inducibility are shown in Fig. 4), although the magnitude of the $T m 5 \mathrm{~b} / \mathrm{Tm} 5 \mathrm{a}$ ratio was different in each cell line. Progestin treatment caused an augmentation in the Tm5b/Tm5a ratio in the T-47DhPRA B8 and E3 clones, as well as the N3 clones shown in Fig. 4A, and in each cell line PRA induction resulted in an abrogation of the progestin effect on the $\mathrm{Tm} 5 \mathrm{~b} / \mathrm{Tm} 5 \mathrm{a}$ ratio, which in E3 and B8 cells was reduced to close to control levels (Fig. 4B and C). No effect of IPTG treatment was observed in the parental lines lacking hPRA inducibility (Fig. 4).

\section{Effect of progestin on distribution of actin-Tm microfilaments in T-47DhPRA cells}

Tm was diffusely expressed in the cytoplasm of control cells and was detected in poorly organized stress fibers. Strongly staining foci or actin-dense protrusions were observed at the periphery of some cells (Fig. 5A). Adhesion belts were not visible and cells displayed a granular appearance. Progestin treatment resulted in increased cell surface, as previously shown in Fig. 1 and fibrous cytoplasmic bundles (Fig. 5E, arrow). No distinct cellular borders were observed between individual cells (Fig. 5C and E). Cells with overexpression of PRA in the absence of progestin treatment had a similar appearance to control cells (Fig. 5, compare B with A). Progestin treatment of PRA-overexpressing cells caused a decrease in fibrous cytoplasmic bundles and a distinctly rounded morphology (Fig. 5D and F). Consistent with the rounded morphology, redistribution of Tm from the cytoplasm to the periphery of cells was noted (Fig. 5F, arrows).

\section{Effect of exogenously transfected Tm5a and $\mathrm{Tm} 5 \mathrm{~b}$ on cell shape in T-47D cells}

The different distribution of Tms in progestintreated cells, depending on the relative levels of PRA and PRB, suggested that the actin-Tm microfilaments may be involved in the cell rounding observed in PRA-overexpressing cells. This possibility was supported by the dependence on PRA levels of the ratios of Tm5a and Tm5b as shown in Fig. 4. To determine whether the alteration in the ratio of $T \mathrm{~m} 5 \mathrm{a}$ and $\mathrm{Tm} 5 \mathrm{~b}$ was implicated in the PRA-mediated changes in cell shape, expression vectors encoding Tm5a or Tm5b were transfected into T-47DhPRA cells. T-47DhPRA cells treated with IPTG to induce PRA, or with vehicle and/or ORG2058, were used as controls and transfected cells were identified by GFP fluorescence. The shape of transfected cells was scored and the proportion of round cells determined. Cells overexpressing PRA and treated with progestin showed an increased proportion of round cells, in line with the decreased surface area shown in Fig. 1, compared with progestin-treated control cells (Fig. 6: compare IPTG+ORG2058+ with IPTG - ORG2058+; $P<0.0001$, one-way ANOVA). There was no statistical difference in the proportion of round cells in control (IPTG - ORG2058 - ), progestin-treated (IPTG ORG2058+) or PRA-overexpressing cells in the absence of ligand (IPTG+ORG2058-). Transfection with Tm5a, in the absence of PRA overexpression, resulted in the same proportion of round cells as observed in the progestin-treated PRAoverexpressing cells (Fig. 6: compare Tm5a with IPTG+ORG2058+; $P=0.41$ one-way ANOVA), demonstrating that expression of Tm5a resulted in an effect on cell rounding that was similar to that provoked by progestin treatment in PRAoverexpressing cells. The proportion of round cells was significantly greater in Tm5a-transfected 
A

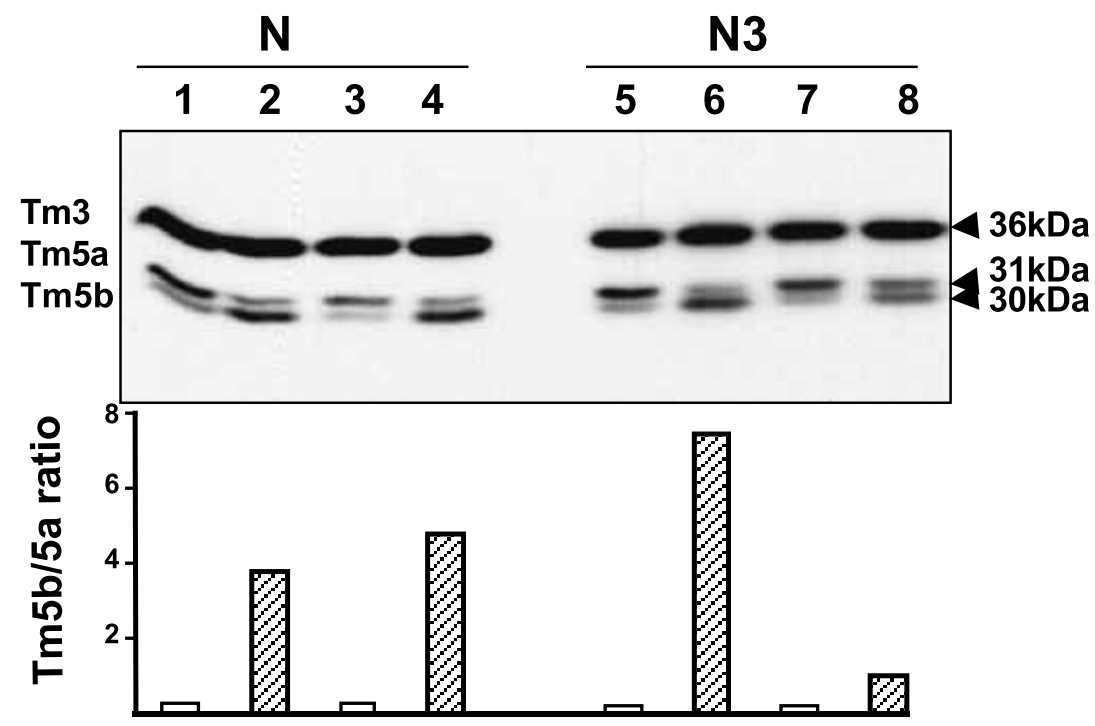

B

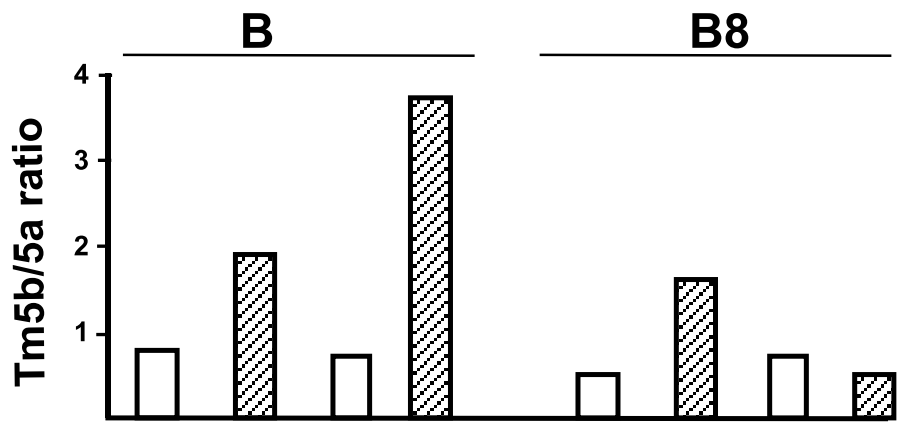

C

E

E3
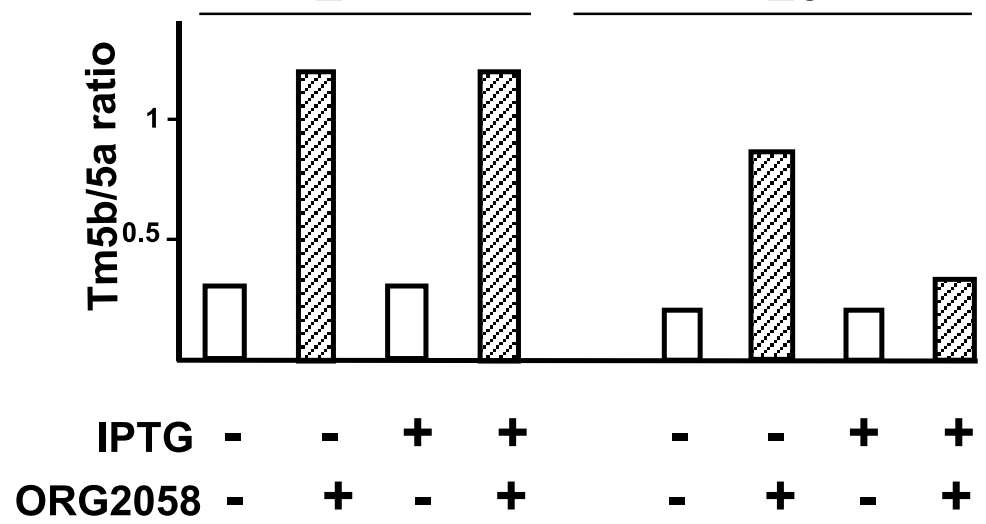

Figure 4 Progestin effects on the expression of TM-5a and TM-5b in T-47D and T-47DhPRA cells. T-47D (-N, -B and -E) parental clones containing the p3'SS vector encoding the lac repressor and T-47DhPRA (-N3, -B8 and -E3) clones containing both the p3'SS and inducible pOP13 hPRA plasmids were treated with IPTG (10 mM) or vehicle. After $24 \mathrm{~h}$ cells were treated with ORG2058 $(10 \mathrm{nM})$ or vehicle, harvested $72 \mathrm{~h}$ later and total cytosol protein prepared. Tm isoform expression was detected using a polyclonal antibody which detects all $\alpha$-TM isoforms on immunoblots $(25 \mu \mathrm{g}$ cytosol protein/lane). Tm5b/Tm5a ratio was calculated densitometrically and presented as histograms. (A) Upper panel, immunoblot of $-\mathrm{N}$ and $-\mathrm{N} 3$ cells, lower panel, histogram of Tm5b/Tm5a ratio. (B and $\mathrm{C}$ ) Histogram of Tm5b/Tm5a ratio in cell lines, $-\mathrm{B}$ and $-\mathrm{B} 8$ and $-\mathrm{E}$ and $-\mathrm{E} 3$. 

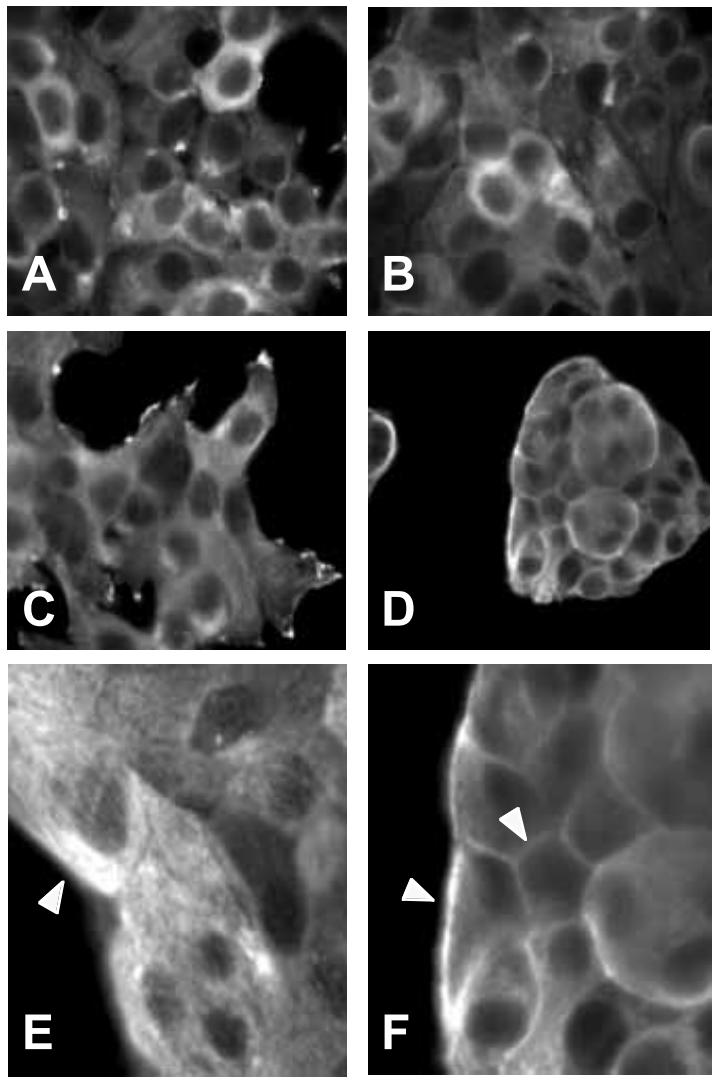

Figure 5 Effects of progestin on Tm distribution in T-47DhPRA cells. T-47DhPRA cells were treated with IPTG (10 mM) or vehicle for $24 \mathrm{~h}$ and treated with ORG2058 (10 nM) or vehicle. Cells were fixed at $72 \mathrm{~h}$, immunolabeled with the ws $\alpha_{f} 9 d$ antibody and the distribution of the Tm isoforms observed. Original magnification $\times 400(A-D)$ or $\times 1000(E$ and $F)$. (A) Vehicle; (B) IPTG (10 mM); (C and E) ORG2058 (10 nM); (D and F) IPTG (10 mM) and ORG2058 (10 nM).

cells than controls (Fig. 6: compare Tm5a with IPTG - ORG2058 - ; $\quad P<0.002, \quad$ one-way ANOVA). Transfection of Tm5b did not have an effect on the proportion of round cells (Fig. 6: compare Tm5b with IPTG - ORG2058 - ; $P=0 \cdot 98$, one-way ANOVA).

\section{Distribution of ezrin in T-47DhPRA cells}

The closely related ezrin-radixin-moesin (ERM) proteins function as general cross-linkers between actin filaments and the cell membrane and are implicated in regulation of cell morphology, adhesion and motility (Tsukita \& Yonemura 1999).

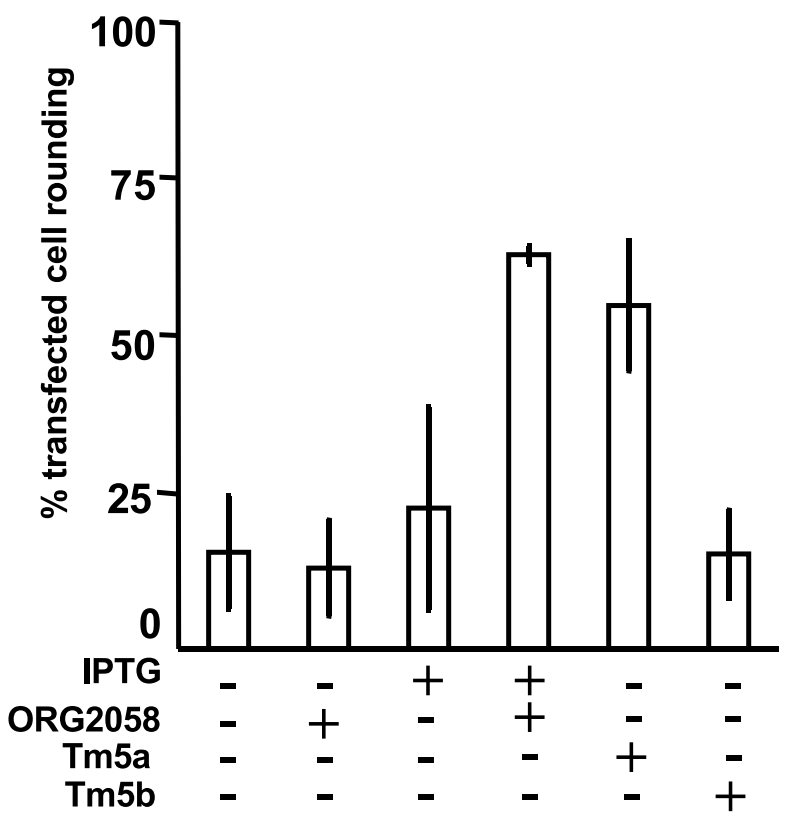

Figure 6 Transfection of Tm5a and Tm5b into T-47DhPRA cells. T-47DhPRA cells were co-transfected with pHßApr-4Tm5a $(1 \mu \mathrm{g})$ and pEGFP $(500 \mathrm{ng})$, or pHßApr-4Tm5b $(1 \mu \mathrm{g})$ and pEGFP $(500 \mathrm{ng})$.

T-47DhPRA cells treated with IPTG $(10 \mathrm{mM})$ or vehicle, transfected with pEGFP $(500 \mathrm{ng})$ and treated with ORG2058 (10 nM) or vehicle were used as controls. Changes in cell shape were estimated by counting the proportion of fluorescent-stained cells that were round over 20 fields att $\times 400$ magnification. Experiments were performed in triplicate and the data expressed as means \pm S.E.M.

Ezrin was the principal ERM protein detected in T-47D cells and low levels only of radixin and moesin were detectable immunohistochemically (data not shown), so ezrin detection formed the focus of ERM protein analysis in this study. No change in ezrin protein levels was observed on immunoblot analysis (Fig. 3) in progestin-treated T-47D cells with or without PRA overexpression, and immunohistochemical analysis to detect ezrin was carried out to document cell distribution of this molecule in progestin-treated cells. In the absence of progestin, ezrin protein staining was evident in densely staining foci at the periphery of cells in control and PRA-overexpressing cells (Fig. 7A and B). Some cytoplasmic foci were also present. Intracellular localization was confirmed after confocal microscope analysis of the fluorescently labeled sections (not shown). Progestin treatment 


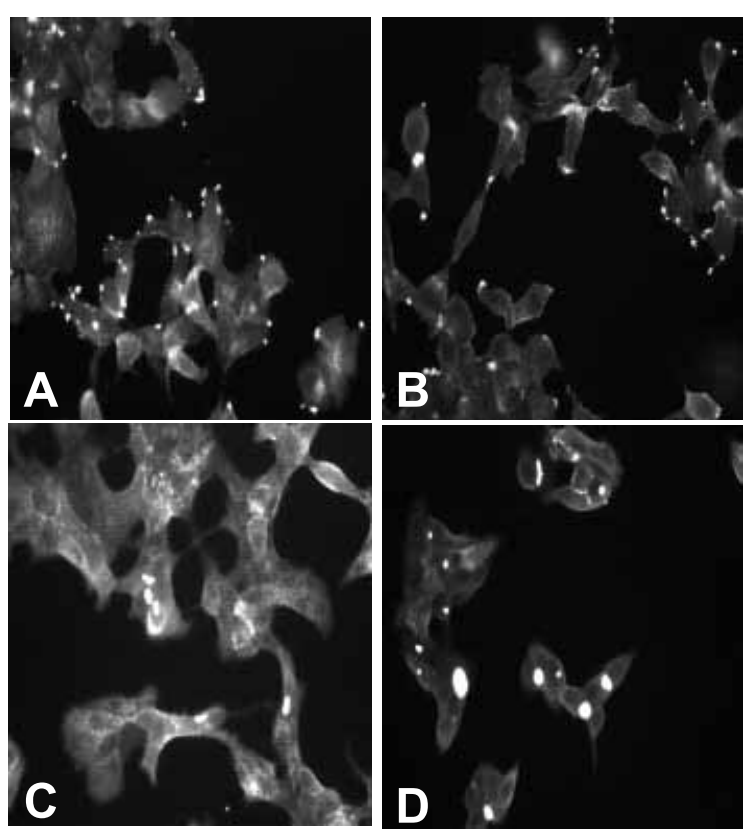

Figure 7 Effects of progestin on ezrin distribution in T-47DhPRA cells. T-47DhPRA cells were treated with IPTG $(10 \mathrm{mM})$ or vehicle. After $24 \mathrm{~h}$ cells were treated with ORG2058 (10 nM) or vehicle and fixed $72 \mathrm{~h}$ thereafter, then stained with a rabbit polyclonal anti-ezrin antibody. (A) vehicle; (B) IPTG (10 mM); (C) ORG2058 (10 nM); (D) IPTG (10 mM) and ORG2058 $(10 \mathrm{nM})$. The data are representative of the same results obtained both in repeated experiments with the same cell line and in separate experiments with a range of T-47DhPRA clones.

resulted in a redistribution of ezrin in control cells, being detected as a marked loss of focal staining at the cell periphery and an increase in cytoplasmic staining (Fig. 7G). Progestin treatment of cells overexpressing PRA resulted in ezrin distribution that was different from that seen either in progestin-treated cells or in control cells. Ezrin was located in discrete foci within the cytoplasm (Fig. 7D). Taken together, the results show that ezrin distribution is under progestin control in T-47D cells and that overexpression of PRA is associated with cytoplasmic focal localization. Given the role of ERM proteins in anchoring stress fibers to the membrane (Tsukita \& Yonemura 1999), the sequestration of ezrin into large cytoplasmic foci only in cells overexpressing PRA suggests that their rounded cell morphology could result in part from the changed localization of ezrin in these cells.

\section{Discussion}

Ovarian hormones are involved in cancer development and progression and there is evidence that disruption in progesterone signaling, through altered expression of PR isoforms, is an early event in cancer development and leads to aberrant regulation of cell morphology. This study has demonstrated that altering the cellular ratio of PRA and PRB, to mimic the increased relative levels of PRA observed in cancers in vivo, markedly changed the nature of progestin regulation of cell morphology.

To determine the mechanisms through which progestin effects on cell morphology were mediated, levels and distribution were investigated initially of membrane-associated and cytoplasmic adhesion components. Overall, steady-state levels of membrane adhesion proteins, $\beta$-catenin and E-cadherin (not shown) either were unchanged by progestin treatment, or, in the case of the focal adhesion protein vinculin, were similarly regulated by progestin treatment in control and PRAoverexpressing cells. Integrin levels were similarly regulated by progestin in control and PRAoverexpressing cells (E M McGowan, S Saad, L J Bendall, K F Bradstock and C L Clarke, unpublished observations). In cell lines with similar levels of PRA and PRB, there is evidence of increased cell spreading and focal adhesions after progestin treatment (Lin et al. 2000, 2001) and this study provides support for progestin regulation of various membrane-associated and cytoplasmic adhesion components. However, as the effects of progestin on membrane components of cell adhesions were the same in cells overexpressing PRA, the differences in progestin effects on cell morphology in cells overexpressing PRA are unlikely to reside primarily at the membrane. The data presented in this study did not exhaustively examine cell distribution and activation states of all of these components, and it may be that there are PRA-specific effects on phosphorylation or cell distribution of some membrane-associated and cytoplasmic adhesion components. This possibility will be explored in future investigations.

Progestin regulation of cell morphology was shown to involve actin microfilaments and evidence for this was provided by the effects of progestin on Tm isoforms. Relative levels of Tm5a and Tm5b were hormonally regulated: in progestin-treated 
control cells Tm5b was elevated at the expense of Tm5a whereas in PRA-overexpressing cells Tm5a and Tm5b were expressed at similar levels. Selective degradation of the Tm isoforms could account for this observation. However, as these Tm isoforms are alternatively spliced products of the same gene (Goodwin et al. 1991), these data suggest that PRA overexpression decreases the progestinmediated elevation in Tm5b and moreover suggest the previously unsuspected progestin sensitivity of the Tm splicing process. The regulation of $\mathrm{Tm}$ splice site selection is not understood although certain factors that appear to mediate alternate splicing in Tm genes have been described (Wieczorek 1988), and alternate Tm splicing has been shown to occur in response to nerve growth factor treatment (Weinberger et al. 1993) or in response to serum factors during myogenesis (Wieczorek 1988).

Transfection of Tm5a and Tm5b isoforms showed that disruption of the levels of these proteins alone was sufficient to mimic the progestin-mediated effects in PRA-overexpressing cells. Introduction of Tm5a provoked cell rounding in most of the transfected cells, in the absence of any hormonal treatment. That cell rounding was also seen upon progestin treatment of cells overexpressing PRA, but not control cells, implicates the Tm5 isoforms in the cell rounding observed in PRA-overexpressing cells and this is further supported as mentioned above by the differential effects of progestin on Tm5 isoform levels seen on immunoblot analysis in PRAoverexpressing vs wild-type cells. The mechanisms underlying the hormonal regulation demonstrated in this study are unclear at present, but progestin regulation of $\alpha$-Tm5 gene splice site selection, taken together with the known role of Tms in actin microfilament regulation (Gunning et al. 1998a), provides support for direct progestin regulation of actin filament dynamics in hormone-dependent cells.

The involvement of actin microfilaments in the cell morphology changes seen upon PRA overexpression was supported also by the altered localization of ezrin in PRA-overexpressing cells. While no changes in ezrin levels were observed on immunoblot analysis, it was clear that progestin treatment altered ezrin localization and moreover that PRA overexpression was associated with a different effect to that seen in control cells. Whereas progestin treatment in control cells caused a reduction in membrane staining of ezrin and increased cytoplasmic granular staining, ezrin localization in PRA-overexpressing cells was in distinct cytoplasmic foci. Current evidence suggests that ezrin anchoring to the membrane via CD44 is required for stress fiber attachment (Vaheri et al. 1997); the sequestration of ezrin in cytoplasmic foci in PRA-overexpressing cells suggests that this protein is unavailable for interaction with the cytoskeleton and is consistent with the relative lack of stress fiber-associated microfilaments in these cells. In contrast to the ezrin, levels of the focal contact protein vinculin were increased in PRAoverexpressing cells despite the decrease in cell surface area which may imply a decrease in focal adhesions. The implication is that vinculin is no longer attached to adhesion sites and that different cellular mechanisms control its targeting and expression when PRA is overexpressed. This study suggests therefore that multiple PRA-sensitive mechanisms appear to exist in order to regulate the expression and localization of cytoskeletalassociated proteins.

The mechanisms of progestin regulation of the cytoskeletal components investigated in this study are not known. Progestin is likely to be acting indirectly on membrane proteins such as ezrin, which were redistributed upon progestin treatment, without alteration in its cellular levels. Progestin effects on vinculin involved increased levels of the protein after prolonged treatment, which may imply transcriptional regulation of vinculin by indirect means. As discussed above, the mechanism of progestin regulation of Tm splice site selection is still to be determined although the delayed appearance of the progestin effect on Tm5 isoform levels suggests that it is likely to be indirect. Although delineation of the mechanisms of PR activity on the cytoskeletal pathways investigated here were beyond the scope of the current study, it is possible that PR modulation of previously described cell signaling pathways in the cytoplasm will be involved. PR, through interaction with $\mathrm{SH} 3$ domain of Src kinases, is able to activate MAP kinase and exert rapid regulation of cytoplasmic signaling pathways (Boonyaratanakornkit et al. 2001) although the delayed appearance of the effects documented in this study may argue against the involvement of a rapid MAP kinase-mediated mechanism. Nevertheless, the results of this study 
support a cytoplasmic action for PR, and delineation of the mechanisms and the relative contributions of nuclear and cytoplasmic PR action in regulation of the cytoskeleton remains the focus of ongoing studies.

Altered regulation of the cytoskeleton upon PRA overexpression is consistent with the changed PRA expression demonstrated in cancers. Progression to malignancy in breast and endometrium is accompanied by disrupted PRA and PRB expression, and a significant proportion of carcinomas express a predominance of one isoform, usually PRA (Graham et al. 1995b, Arnett-Mansfield et al. 2001, Mote et al. 2002). This is associated with poor clinical outcomes: in endometrial cancer, PRA predominance is significantly more common in tumors of higher grade, indicating an association between PR isoform predominance and poor prognosis (Arnett-Mansfield et al. 2001). This study suggests that PRA predominance may confer progestin responsiveness on specific components of the cytoskeleton and shows for the first time that the PR isoforms have distinct effects on the cytoskeleton by regulating multiple signaling pathways, some of which may be associated with changes in the cytoskeleton known to be associated with cancer development and progression.

\section{Acknowledgements}

RU38486 was a kind gift of $\mathrm{J}$ P Raynaud, Roussel-Uclaf, Romainville, France. The authors thank Nicole Bryce for construction of the Tm5a and Tm5b plasmids and Claire Stockwell for technical assistance. This study was supported by the National Health and Medical Research Council of Australia and the Leo and Jenny Leukaemia Foundation. E M M received a Dora Lush Biomedical Research Scholarship from the National Health and Medical Research Council of Australia. J A I H was supported by a Faculty of Medicine Postgraduate Scholarship from the University of Sydney.

\section{References}

Alexander IE, Clarke CL, Shine J \& Sutherland RL 1989 Progestin inhibition of progesterone receptor gene expression in human breast cancer cells. Molecular Endocrinology 3 1377-1386.

Arnett-Mansfield RL, de Fazio A, Wain GV, Jaworski RC, Byth K, Mote PA \& Clarke CL 2001 Relative expression of progesterone receptors $\mathrm{A}$ and $\mathrm{B}$ in endometrioid cancers of the endometrium. Cancer Research 61 4576-4582.
Boonyaratanakornkit V, Scott MP, Ribon V, Sherman L, Anderson SM, Maller JL, Miller WT \& Edwards DP 2001 Progesterone receptor contains a proline-rich motif that directly interacts with SH3 domains and activates c-src family tyrosine kinases. Molecular Cell 8 1-20.

Clarke CL, Zaino RJ, Feil PD, Miller JV, Steck ME, Ohlsson-Wilhelm BM \& Satyaswaroop PG 1987 Monoclonal antibodies to human progesterone receptor: characterization by biochemical and immunohistochemical techniques. Endocrinology $1211123-1132$

David-Pfeuty T \& Singer SJ 1980 Altered distribution of the cytoskeletal proteins vinculin and alpha-actinin in cultured fibroblasts transformed by Rous sarcoma virus. PNAS 77 6687-6691.

Edelman GM \& Yahara I 1976 Temperature-sensitive changes in surface modulating assemblies of fibroblasts transformed by mutants of Rous sarcoma virus. PNAS 73 2047-2051.

Goodwin LO, Lees-Miller JP, Leonard MA, Cheley SB \& Helfman DM 1991 Four fibroblast tropomyosin isoforms are expressed from the rat alpha-tropomyosin gene via alternative RNA splicing and the use of two promoters. Fournal of Biological Chemistry 266 8408-8415.

Graham J, Roman S, McGowan E, Sutherland R \& Clarke C 1995a Preferential stimulation of human progesterone receptor $\mathrm{B}$ expression by estrogen in T-47D human breast cancer cells. Fournal of Biological Chemistry 270 30693-30700.

Graham JD, Yeates C, Balleine RL, Harvey SS, Milliken JS, Bilous AM \& Clarke CL $1995 b$ Progesterone receptor A and B protein expression in human breast cancer. Fournal of Steroid Biochemistry and Molecular Biology 56 93-98.

Gronemeyer H 1991 Transcription activation by estrogen and progesterone receptors. Annual Review of Genetics 25 89-123.

Gunning P, Hardeman E, Jeffrey P \& Weinberger RP $1998 a$ Creating intracellular structural domains: spatial segregation of actin and tropomyosin isoforms in neurons. BioEssays 20 892-900.

Gunning P, Weinberger R, Jeffrey P \& Hardeman E 1998 b Isoform sorting and the creation of intracellular compartments. Annual Review of Cell and Developmental Biology 14 339-372.

Hall A 1998 Rho GTPases and the actin cytoskeleton. Science $\mathbf{2 7 9}$ 509-514.

Hovland AR, Powell RL, Takimoto GS, Tung L \& Horwitz KB 1998 An N-terminal inhibitory function, IF, suppresses transcription by the A-isoform but not the B-isoform of human progesterone receptor. Fournal of Biological Chemistry $\mathbf{2 7 3}$ 5455-5460.

Kastner P, Krust A, Turcotte B, Stropp U, Tora L, Gronemeyer H \& Chambon P 1990 Two distinct estrogen-regulated promoters generate transcripts encoding the two functionally different human progesterone receptor forms A and B. EMBO fournal 9 $1603-1614$.

Keydar I, Chen L, Karby S, Weiss FR, Delarea J, Radu M, Chaitcik S \& Brenner HJ 1979 Establishment and characterization of a cell line of human breast carcinoma origin. European fournal of Cancer $\mathbf{1 5}$ 659-670.

Leavitt J, Ng SY, Aebi U, Varma M, Latter G, Burbeck S, Kedes L \& Gunning P 1987a Expression of transfected mutant beta-actin genes: alterations of cell morphology and evidence for autoregulation in actin pools. Molecular and Cellular Biology 7 $2457-2466$.

Leavitt J, Ng SY, Varma M, Latter G, Burbeck S, Gunning P \& Kedes L 1987b Expression of transfected mutant beta-actin genes: transitions toward the stable tumorigenic state. Molecular and Cellular Biology 7 2467-2476.

Lin VCL, Ng EH, Aw SE, Tan MGK, Ng EHL \& Bay BH 2000 Progesterone induces focal adhesion in breast cancer cells MDAMB-231 transfected with progesterone receptor complementary DNA. Molecular Endocrinology 14 348-357. 
Lin VCL, Eng AS, Hen NE, Ng EH \& Chowdhury SH 2001 Effect of progesterone on the invasive properties and tumor growth of progesterone receptor-transfected breast cancer cells MDA-MB231. Clinical Cancer Research 7 2880-2886.

Lydon JP, Ge G, Kittrell FS, Medina D \& O'Malley BW 1999 Murine mammary gland carcinogenesis is critically dependent on progesterone receptor function. Cancer Research 59 4276-4284.

MacMahon B \& Feinlieb M 1960 Breast cancer in relation to nursing and menopausal history. Fournal of the National Cancer Institute 24 733-753.

McDonnell DP, Shahbaz MM, Vegeto E \& Goldman ME 1994 The human progesterone receptor A-form functions as a transcriptional modulator of mineralocorticoid receptor transcriptional activity. Fournal of Steroid Biochemistry and Molecular Biology 48 425-432.

McGowan EM \& Clarke CL 1999 Effect of overexpression of progesterone receptor $\mathrm{A}$ on endogenous progestin-sensitive endpoints in breast cancer cells. Molecular Endocrinology 13 $1657-1671$

Mote PA, Balleine RL, McGowan EM \& Clarke CL 1999 Co-localization of progesterone receptors A and B by dual immunofluorescent histochemistry in human endometrium during the menstrual cycle. Fournal of Clinical Endocrinology and Metabolism 84 2963-2971.

Mote PA, Bartow S, Tran N \& Clarke CL 2002 Loss of co-ordinate expression of progesterone receptors A and $\mathrm{B}$ is an early event in breast carcinogenesis. Breast Cancer Research and Treatment 72 163-172.

Percival JM, Thomas G, Cock T-A, Gardiner EM, Jeffrey PL, Lin JJ-C, Weinberger RP \& Gunning P 2000 Sorting of tropomyosin in synchronised NIH 3T3 fibroblasts: evidence for distinct microfilament populations. Cell Motility and the Cytoskeleton $\mathbf{4 7}$ 189-208.

Pienta KJ, Partin AW \& Coffey DS 1989 Cancer as a disease of DNA organization and dynamic cell structure. Cancer Research 49 2525-2532.

Pollack R, Osborn M \& Weber K 1975 Patterns of organization of actin and myosin in normal and transformed cultured cells. PNAS 72 994-998.

Qin H \& Gunning P 1997 The 3 '-end of the human beta-actin gene enhances activity of the beta-actin expression vector system: construction of improved vectors. Fournal of Biochemical and Biophysical Methods 36 63-72.

Ross BC, Paganini-Hill A, Wan PC \& Pike MC 2000 Effect of hormone replacement therapy on breast cancer risk: estrogen versus estrogen plus progesterone. Fournal of the National Cancer Institute 92 328-332.

Schevzov G, Gunning P, Jeffrey PL, Temm-Grove C, Helfman DM, Lin JJ-C \& Weinberger R 1997 Tropomyosin localization reveals distinct populations of microfilaments in neurites and growth cones. Molecular and Cellular Neurosciences 8 439-454.

Takahashi K, Heine UI, Junker JL, Colburn NH \& Rice JM 1986 Role of cytoskeletal changes and expression of the H-ras oncogene during promotion of neoplastic transformation in mouse epithelial JB6 cells. Cancer Research 46 5923-5932.

Tsukita S \& Yonemura S 1999 Cortical actin organization: lessons from ERM (ezrin/radixin/moesin) proteins. Fournal of Biological Chemistry 274 34507-34510.

Tung L, Mohamed MK, Hoeffler JP, Takimoto GS \& Horwitz KB 1993 Antagonist-occupied human progesterone B-receptors activate transcription without binding to progesterone response elements and are dominantly inhibited by A-receptors. Molecular Endocrinology 7 1256-1265.

Vaheri A, Carpen O, Heiska L, Helander TS, Jaaskelainen J, Majander-Nordenswan P, Sainio M, Timonen T \& Turunen O 1997 The ezrin protein family: membrane-cytoskeleton interactions and disease associations. Current Opinion in Cell Biology 9 659-666.

Vegeto E, Shahbaz MM, Wen DX, Goldman ME, O'Malley BW \& McDonnell DP 1993 Human progesterone receptor A form is a cell- and promoter-specific repressor of human progesterone receptor B function. Molecular Endocrinology 7 $1244-1255$.

Weinberger RP, Henke RC, Tolhurst O, Jeffrey PL \& Gunning P 1993 Induction of neuron-specific tropomyosin mRNAs by nerve growth factor is dependent on morphological differentiation. Fournal of Cell Biology 120 205-215.

Wieczorek DF 1988 Regulation of alternatively spliced alphatropomyosin gene expression by nerve extract. Fournal of Biological Chemistry 263 10456-10463.

Writing Group for the Women's Health Initiative Investigators 2002 Risks and benefits of estrogen plus progestin in healthy postmenopausal women: principal results from the women's health initiative randomized controlled trial. Fournal of the American Medical Association $\mathbf{2 8 8} 321-333$.

Received 28 March 2003 Accepted 5 June 2003 